PROCEEDINGS OF THE

AMERICAN MATHEMATICAL SOCIETY

Volume 128, Number 5, Pages 1307-1312

S 0002-9939(99)05130-8

Article electronically published on October 18, 1999

\title{
GENERALIZED MATLIS DUALITY
}

\author{
RICHARD G. BELSHOFF, EDGAR E. ENOCHS, AND JUAN RAMON GARCÍA ROZAS
}

(Communicated by Wolmer V. Vasconcelos)

\begin{abstract}
Let $R$ be a commutative noetherian ring and let $E$ be the minimal injective cogenerator of the category of $R$-modules. A module $M$ is said to be reflexive with respect to $E$ if the natural evaluation map from $M$ to $\operatorname{Hom}_{R}\left(\operatorname{Hom}_{R}(M, E), E\right)$ is an isomorphism. We give a classification of modules which are reflexive with respect to $E$. A module $M$ is reflexive with respect to $E$ if and only if $M$ has a finitely generated submodule $S$ such that $M / S$ is artinian and $R / \operatorname{ann}(M)$ is a complete semi-local ring.
\end{abstract}

Matlis and Gabriel in 7] and [5] considered modules over a complete local ring $R$. They showed that if the dual of an $R$-module is taken with respect to $E_{R}(k)$ (the injective envelope of the residue field $k$ of $R$ ), then finitely generated and artinian modules are reflexive.

Various authors have considered related questions. For example, dropping the condition that $R$ be complete or weakening local to semilocal ([1, [2, [6], [8, 9]).

In this paper we let $R$ be any commutative noetherian ring and let $E$ be the minimal injective cogenerator of the category of $R$-modules. We give a classification of modules which are reflexive with respect to $E$. The result is that a module $M$ is reflexive with respect to $E$ if and only if $M$ has a finitely generated submodule $S$ such that $M / S$ is artinian and if $R / I$ is a complete semilocal ring where $I=$ $\operatorname{ann}(M)$.

We denote by $\Omega$ the maximal spectrum of $R$, and we let $E=\bigoplus_{m \in \Omega} E_{R}(R / m)$ be the minimal injective cogenerator in the category of $R$-modules. For an $R$-module $M$ we let $M^{\vee}=\operatorname{Hom}_{R}(M, E)$ and call $M^{\vee}$ the Matlis dual of $M$. If the canonical map $M \rightarrow M^{\vee \vee}$ is an isomorphism we say that $M$ is (Matlis) reflexive. We note that for any $M$, the map $M \rightarrow M^{\vee \vee}$ is an injection. From this it is easy to conclude that $\operatorname{ann}(M)=\operatorname{ann}\left(M^{\vee}\right)$.

If $S \subset R$ is a multiplicative set and the canonical map $M \rightarrow S^{-1} M$ is an isomorphism, we write $M=S^{-1} M$. If $M=S^{-1} M$, then also $M^{\vee}=S^{-1}\left(M^{\vee}\right)$. When $S=R-P$ is the complement of a prime ideal $P$ of $R$ we use the usual notation $M_{P}$.

If $R$ is a local ring we let $\hat{R}$ denote its completion. If $M$ is finitely generated we note that $\hat{R} \otimes_{R} M \cong \hat{M}$ (the completion of $\left.M\right)$. We write $\hat{R} \otimes_{R} M \cong \hat{M}=M$ to mean that $M \rightarrow \hat{R} \otimes_{R} M \cong \hat{M}$ is an isomorphism.

We note that if $m \in \Omega$ and $M$ is a finitely generated $R$-module $M$, then $\operatorname{Hom}_{R}(M, E(R / m)) \neq 0$ if and only if $\operatorname{ann}(M) \subset m$.

Received by the editors January 28, 1998 and, in revised form, July 1, 1998.

1991 Mathematics Subject Classification. Primary 13C05; Secondary 13 H99.

Key words and phrases. Matlis, duality. 
If $R$ is a complete local ring, then all finitely generated and all artinian $R$-modules are reflexive. If $R$ is a complete semilocal ring, then since $R$ is the product of a finite number of complete local rings, we still have finitely generated and artinian modules over $R$ are reflexive.

Lemma 1. If $I \subset R$ is an ideal and $I M=0$ for an $R$-module $M$, then $M$ is reflexive as an $R$-module if and only if $M$ is reflexive as an $R / I$-module.

Proof. This follows from the fact that $\operatorname{Hom}_{R}(R / I, E)$ is a minimal injective cogenerator over $R / I$ and that, since $M \otimes_{R} R / I \cong M$, we have

$$
\operatorname{Hom}_{R / I}\left(M, \operatorname{Hom}_{R}(R / I, E)\right) \cong \operatorname{Hom}_{R}(M, E) .
$$

Lemma 2. If $S \subset R$ is multiplicative and $S^{-1} M=M$ for an $R$-module $M$, then $M$ is reflexive as an $R$-module if and only if $M$ is reflexive as an $S^{-1} R$-module.

Proof. As in Lemma 1 $\operatorname{Hom}_{R}\left(S^{-1} R, E\right)$ is a minimal injective cogenerator over $S^{-1} R$ and

$$
\operatorname{Hom}_{S^{-1} R}\left(M, \operatorname{Hom}_{R}\left(S^{-1} R, E\right)\right) \cong \operatorname{Hom}_{R}(M, E) .
$$

The following result strengthens Theorem 2(i) in [1].

Theorem 3. Let $R$ be a local ring and $M$ a finitely generated $R$-module. Then $M$ is reflexive if and only if $\hat{R} \otimes_{R} M=M$.

Proof. For any such $M$ we have the commutative diagram

$$
\begin{array}{cll}
M & \longrightarrow & \operatorname{Hom}_{R}\left(\operatorname{Hom}_{R}\left(M, E_{R}(k)\right), E_{R}(k)\right) \\
\downarrow & \downarrow & \\
\hat{M}=\hat{R} \otimes_{R} M & \longrightarrow & \operatorname{Hom}_{\hat{R}}\left(\operatorname{Hom}_{\hat{R}}\left(\hat{M}, E_{\hat{R}}(k)\right), E_{\hat{R}}(k)\right)
\end{array}
$$

where $k$ is the residue field of $R$ and of $\hat{R}$. The bottom horizontal arrow is an isomorphism since $\hat{M}$ is a reflexive $\hat{R}$-module.

But we claim that the right vertical arrow is an isomorphism. For $E_{R}(k)=E_{\hat{R}}(k)$ and

$$
\begin{gathered}
\operatorname{Hom}_{\hat{R}}\left(\hat{M}, E_{R}(k)\right)=\operatorname{Hom}_{\hat{R}}\left(\hat{R} \otimes_{R} M, E_{R}(k)\right) \\
=\operatorname{Hom}_{R}\left(M, \operatorname{Hom}_{\hat{R}}\left(\hat{R}, E_{R}(k)\right)\right)=\operatorname{Hom}_{R}\left(M, E_{R}(k)\right) .
\end{gathered}
$$

Since $A=\operatorname{Hom}_{R}\left(M, E_{R}(k)\right)$ and $E_{R}(k)$ are artinian $R$ and $\hat{R}$-modules, we have $\operatorname{Hom}_{\hat{R}}\left(A, E_{R}(k)\right)=\operatorname{Hom}_{R}\left(A, E_{R}(k)\right)$. It follows that $M \rightarrow \hat{R} \otimes_{R} M$ is an isomorphism if and only if $M$ is reflexive.

Corollary 4. If $I$ is an ideal in a local ring $R$, then $R / I$ is reflexive as an $R$-module (or as an $R / I$-module) if and only if $R / I$ is a complete local ring.

Proof. This follows from the fact that $\hat{R} \otimes_{R} R / I=\widehat{R / I}$.

Lemma 5. If $M$ is an $R$-module and $S \subset M$ is a submodule, then $M$ is reflexive if and only if $S$ and $M / S$ are reflexive. 
Proof. The commutative diagram

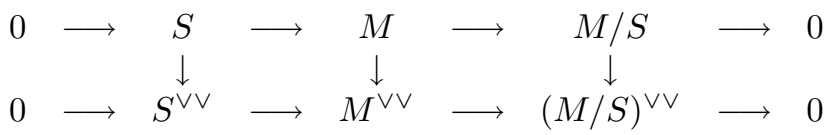

has exact rows and the vertical maps are all injections. So the result follows.

Lemma 6. No infinite direct sum of nonzero $R$-modules is reflexive.

Proof. Let $\bigoplus_{i \in I} M_{i}$ be reflexive where $I$ is infinite and $M_{i} \neq 0$ for all $i \in I$. We have $\left(\bigoplus_{i \in I} M_{i}\right)^{\vee}=\prod_{i \in I}\left(M_{i}^{\vee}\right)$, and the canonical map

$$
\bigoplus_{i \in I} M_{i} \rightarrow\left(\prod_{i \in I}\left(M_{i}^{\vee}\right)\right)^{\vee} \cong\left(\bigoplus_{i \in I} M_{i}\right)^{\vee \vee}
$$

sends $\left(x_{i}\right)_{i \in I}$ to the map $\left(\phi_{i}\right)_{i \in I} \mapsto \sum_{i \in I} \phi_{i}\left(x_{i}\right)$. So the image of $\left(x_{i}\right)_{i \in I}$ is 0 on $\bigoplus_{i \in I}\left(M_{i}^{\vee}\right) \subset \prod_{i \in I}\left(M_{i}^{\vee}\right)$ if and only if $x_{i}=0$ for all $i$, i.e., if and only if $\left(x_{i}\right)_{i \in I}=0$. But $\bigoplus_{i \in I}\left(M_{i}^{\vee}\right) \neq \prod_{i \in I}\left(M_{i}^{\vee}\right)$ so there is a nonzero linear $f: \prod_{i \in I}\left(M_{i}^{\vee}\right) \rightarrow E$ which is 0 on $\bigoplus_{i \in I}\left(M_{i}^{\vee}\right)$. By the above no such $f$ can be the image of an $\left(x_{i}\right)_{i \in I} \in \bigoplus_{i \in I} M_{i}$ and so $\bigoplus_{i \in I} M_{i}$ is not reflexive.

Corollary 7. If $M$ is reflexive there is a finitely generated submodule $S$ such that $M / S$ is artinian.

Proof. If $M=0$ this is trivial. If $M \neq 0$ there is a finitely generated $S_{1} \subset M$ such that $\operatorname{Soc}\left(M / S_{1}\right) \neq 0$. If $\operatorname{Soc}\left(M / S_{1}\right)$ is essential in $M / S_{1}$, it is well known that $M / S_{1}$ (and in fact $\left.E\left(M / S_{1}\right)\right)$ is artinian. If it is not essential, let $N / S_{1} \cap \operatorname{Soc}\left(M / S_{1}\right)=0$ with $S_{1} \subset N, S_{1} \neq N$. Then there is a finitely generated $S_{2}$ with $S_{1} \subset S_{2} \subset N$ and $\operatorname{Soc}\left(N / S_{2}\right) \neq 0$. But then $\operatorname{Soc}\left(M / S_{1}\right) \rightarrow \operatorname{Soc}\left(M / S_{2}\right)$ is injective but not surjective. We repeat the procedure and see that it must stop, for otherwise if $T=\bigcup S_{n}$, then $\operatorname{Soc}(M / T)$ is an infinite direct sum. This is impossible by Lemma 6 ,

The argument above is taken from Enochs [3, Proposition 1.3, and is included here for completeness.

Proposition 8. Let $M$ be an $R$-module and suppose that for some $m \in \Omega$, $\operatorname{Hom}_{R}(M, E(R / n))=0$ for $n \in \Omega, n \neq m$. Then if $M$ is reflexive, $M_{m}=M$ and $\operatorname{Hom}_{R}\left(M^{\vee}, E(R / n)\right)=0$ for $n \neq m$.

Proof. Let $M$ be reflexive and $m \in \Omega$ be such that $\operatorname{Hom}_{R}(M, E(R / n))=0$ for $n \neq m$. If $M \neq 0$, we have a natural nonzero homomorphism $M \rightarrow M^{\vee \vee}=$ $\operatorname{Hom}_{R}\left(M^{\vee}, E\right)(n \in \Omega)$. So if $\operatorname{Hom}_{R}\left(M^{\vee}, E(R / n)\right) \neq 0$, then the projection $E \rightarrow$ $E(R / n)$ induces $\operatorname{Hom}_{R}\left(M^{\vee}, E\right) \rightarrow \operatorname{Hom}_{R}\left(M^{\vee}, E(R / n)\right)$ and so we have a nonzero homomorphism $M \rightarrow \operatorname{Hom}_{R}\left(M^{\vee}, E(R / n)\right)$. So then

$$
\operatorname{Hom}_{R}\left(M, \operatorname{Hom}_{R}\left(M^{\vee}, E(R / n)\right)\right) \neq 0 \text {. }
$$


But

$$
\operatorname{Hom}_{R}\left(M, \operatorname{Hom}_{R}\left(M^{\vee}, E(R / n)\right)\right) \cong \operatorname{Hom}_{R}\left(M^{\vee}, \operatorname{Hom}_{R}(M, E(R / n))\right)
$$

so $\operatorname{Hom}_{R}(M, E(R / n)) \neq 0$. Hence $n=m$.

Since $M^{\vee}=\operatorname{Hom}_{R}(M, E(R / m))$, it follows easily using properties of $E(R / m)$ that $\left(M^{\vee}\right)_{m}=M^{\vee}$. But then $\left(M^{\vee \vee}\right)_{m}=M^{\vee \vee}$ and so $M_{m}=M$. It now follows by Lemma 2 that $M$ is a reflexive $R_{m}$-module as well as a reflexive $R$-module.

In the next result we use the fact that if $A$ is an artinian $R$-module, then there are distinct maximal ideals $n_{1}, n_{2}, \ldots, n_{t} \in \Omega$ and a decomposition

$$
A=A_{1} \oplus A_{2} \oplus \cdots \oplus A_{t}
$$

such that $\left(A_{i}\right)_{n_{i}}=A_{i}$. The $n_{1}, \ldots, n_{t}$ and the decomposition are unique in the obvious sense. If $B \subset A$ is a submodule, then

$$
B=\left(B \cap A_{1}\right) \oplus\left(B \cap A_{2}\right) \oplus \cdots \oplus\left(B \cap A_{t}\right)
$$

gives the corresponding decomposition of $B$. We note that $\operatorname{Hom}_{R}\left(A_{i}, E(R / m)\right)=0$ for $m \in \Omega, m \notin\left\{n_{1}, \ldots, n_{t}\right\}$.

Theorem 9. Let $M$ be a finitely generated $R$-module and let $I=\operatorname{ann}(M)$. Then $M$ is reflexive if and only if $R / I$ is a complete semilocal ring.

Proof. We have $M^{\vee}=\operatorname{Hom}_{R}(M, \bigoplus E(R / m)) \cong \bigoplus \operatorname{Hom}_{R}(M, E(R / m))$ (the direct sum over all $m \in \Omega$ ) since $M$ is finitely generated. Since $M^{\vee}$ is also reflexive, we see by Proposition 8 that $\operatorname{Hom}_{R}(M, E(R / m))=0$ except for a finite number of $m \in \Omega$. Let $n_{1}, n_{2}, \ldots, n_{t} \in \Omega$ be distinct elements of $\Omega$ such that $\operatorname{Hom}_{R}(M, E(R / m))=$ 0 for $m \notin\left\{n_{1}, n_{2}, \ldots, n_{t}\right\}$, so $M^{\vee}=\bigoplus_{i=1}^{t} \operatorname{Hom}_{R}\left(M, E\left(R / n_{i}\right)\right)$. We can assume $\operatorname{Hom}_{R}\left(M, E\left(R / n_{i}\right)\right) \neq 0$ for $i=1,2, \ldots, t$. If $M_{i}$ denotes $\operatorname{Hom}_{R}\left(M, E\left(R / n_{i}\right)\right)$, then since $\operatorname{Hom}_{R}\left(M_{i}, E(R / m)\right)=0$ for $m \neq n_{i}$, we see $\operatorname{Hom}_{R}\left(R / I_{i}, E(R / m)\right)=0$ for $m \neq n_{i}$ where $I_{i}=\operatorname{ann}\left(M_{i}\right)$. Hence $I_{i}$ is contained in only one maximal ideal, namely $n_{i}$. Hence $R / I_{i}$ is a local ring and $\left(R / I_{i}\right)_{n_{i}}=R / I_{i}$.

Since $R / I_{i}$ is also reflexive and finitely generated, we get that $R / I_{i}$ is a reflexive $R_{n_{i}}$-module by Lemma 2. But then by Theorem 3 , $\widehat{R_{n_{i}}} \otimes_{R_{n_{i}}} R / I_{i}=R / I_{i}$. This means that $R / I_{i}$ is a complete local ring.

But now if $I=\operatorname{ann}(M)$, we have $I=\bigcap_{i=1}^{t} I_{i}$. The $I_{1}, \ldots, I_{t}$ are pairwise comaximal so by the Chinese remainder theorem $R / I \cong R / I_{1} \times R / I_{2} \times \cdots \times R / I_{t}$. Hence $R / I$ is a complete semilocal ring.

Now assume that $M$ is a finitely generated $R$-module and that $R / I$ is a complete semilocal ring where $I=\operatorname{ann}(M)$. Then $M$ is reflexive as an $R / I$-module and so by Lemma 1 is reflexive as an $R$-module.

Corollary 10. If $I \subset R$ is an ideal, then $R / I$ is reflexive if and only if $R / I$ is a complete semilocal ring.

Proof. Immediate.

Corollary 11. If $I, J \subset R$ are ideals such that $R / I$ and $R / J$ are complete semilocal rings, then $R / I J$ is a complete semilocal ring. 
Proof. We only need argue that $R / I J$ is a reflexive $R$-module. We have the exact sequence

$$
0 \rightarrow I / I J \rightarrow R / I J \rightarrow R / I \rightarrow 0 .
$$

But $R / I$ is a reflexive $R$-module, and since $I / I J$ is a quotient of $(R / J)^{n}$ for some $n \geq 1$ it is a reflexive $R$-module. Hence $R / I J$ is a reflexive $R$-module. (These claims all use Lemma [5)

We now give the complete classification of reflexive modules.

Theorem 12. An R-module $M$ is reflexive if and only if it has a finitely generated submodule $S$ such that $M / S$ is artinian and if $R / I$ is a complete semilocal ring where $I=\operatorname{ann}(M)$.

Proof. Assume that $M$ is reflexive. By Corollary 7 we have the finitely generated $S$ with $M / S$ artinian. Since $S$ is reflexive and finitely generated, $R / J$ is a complete semilocal ring where $J=\operatorname{ann}(S)$ by Theorem 9. Since $M / S$ is artinian and reflexive, $(M / S)^{\vee}$ is noetherian, i.e., finitely generated. Hence, if $K=\operatorname{ann}(M / S)^{\vee}$, then $R / K$ is complete semilocal. But $\operatorname{ann}(M / S)^{\vee}=\operatorname{ann}(M / S)$. Since $I=\operatorname{ann}(M) \supset$ $J K$ and since $R / J K$ is complete semilocal by Corollary 11, we see that $R / I$ is complete semilocal.

Conversely assume that $M$ has a finitely generated submodule $S \subset M$ with $M / S$ artinian and that $R / I$ is a complete semilocal ring where $I=\operatorname{ann}(M)$.

Then, since $J=\operatorname{ann}(S) \supset \operatorname{ann}(M)=I$, we see that $R / J$ is complete and semilocal. Hence $S$ is reflexive.

Since $M / S$ is artinian and $K=\operatorname{ann}(M / S) \supset \operatorname{ann}(M)=I$, we see that $R / K$ is complete and semilocal. Then the artinian $R / K$-module is reflexive as an $R / K$ module. By Lemma 1, this implies that $M / S$ is a reflexive $R$-module. But then by an appeal to Lemma 5 it follows that $M$ is a reflexive $R$-module.

Examples. 1. If $k$ is an algebraically closed field and $I \subset k\left[x_{1}, \ldots, x_{n}\right]$ is such that $k\left[x_{1}, \ldots, x_{n}\right] / I$ is semilocal, then in fact $k\left[x_{1}, \ldots, x_{n}\right] / I$ is artinian. Hence a finitely generated reflexive module $M$ over $k\left[x_{1}, \ldots, x_{n}\right]$ has finite length. Since the dual $A^{\vee}$ of an artinian reflexive $k\left[x_{1}, \ldots, x_{n}\right]$-module $A$ is finitely generated, we see that $A$ also has finite length. So by Theorem 12 the reflexive $k\left[x_{1}, \ldots x_{n}\right]$-modules are exactly those of finite length.

2. If $k$ is any field and $R=k[[x]][y]$, then $R /(y)$ is a complete local ring and so is a reflexive $R$-module which is not of finite length.

Remark. We recall that a module $M$ is said to be cotorsion if $\operatorname{xt}^{1}(F, M)=0$ for all flat $R$-modules $F$. In [4, finitely generated cotorsion modules were characterized over a commutative noetherian ring $R$ of finite Krull dimension. In effect, over such a ring $R$, a finitely generated module $M$ is cotorsion if and only if it is reflexive. We do not know if this holds if we allow $R$ to have infinite Krull dimension.

\section{REFERENCES}

[1] R. Belshoff, Some Change of Ring Theorems for Matlis Reflexive Modules, Comm. Algebra 22 (1994), 3545-3552. MR 95h:13010

[2] F.C. Cheng, and M.Y. Wang, Homological Dimension of G-Matlis dual modules over semilocal rings, Comm. Algebra 21 (1993), no. 4, 1215-1220. [MR 94e:13016

[3] E. Enochs, Flat covers and flat cotorsion modules, Proc. Amer. Math. Soc. (2) 92 (1984), 179-184. MR 85j:13016 
[4] E. Enochs, J.R. Garcia Rozas, and L. Oyonarte Finitely generated cotorsion modules, preprint.

[5] P. Gabriel, Objets injectifs dans les categories abeliennes, Seminare Dubriel 12 (1959), no. 17.

[6] P. Jara, and E. Santos, Matlis reflexive modules on complete rings, J. Pure Appl. Algebra 106 (1996), no. 1, 77-91. MR 96j:13014.

[7] E. Matlis, Injective Modules over Noetherian Rings, Pacific J. Math. 8 (1958), 511-528. MR 20:5800

[8] I. Nishitani, A generalized Matlis duality for certain topological rings, Comm. Algebra 22 (1994), no. 7, 2669-2676. MR 95d:16058

[9] M.Y. Wang and G.M. Zhang, Homological properties of generalized Matlis reflexive modules over commutative semilocal rings, Sichuan Shifan Daxue Xuebao Ziran Kexue Ban 16 (1993), no. 1, 14-19. MR 94k:13014

Department of Mathematics, Southwest Missouri State University, Springfield, MisSOURI 65804

E-mail address: belshoff@math.smsu.edu

Department of Mathematics, University of Kentucky, Lexington, Kentucky 40506

E-mail address: enochs@ms.uky.edu

Department of Algebra and Analysis, University of Almería 04120 Almería, Spain

E-mail address: jrgrozas@ualm.es 